\title{
Suppression of the persistent spin Hall current by defect scattering
}

\author{
Jun-ichiro Inoue* \\ Department of Applied Physics, Nagoya University, Nagoya 464-8603, Japan and CREST-JST, Japan \\ Gerrit E. W. Bauer \\ Department of NanoScience, Delft University of Technology, Lorentzweg 1, 2628CJ Delft, The Netherlands \\ Laurens W. Molenkamp \\ Physikalisches Institut (EP3), Universität Würzburg, D-97074 Würzburg, Germany \\ (Received 1 December 2003; revised manuscript received 26 January 2004; published 12 July 2004)
}

\begin{abstract}
We study the linear response spin Hall conductivity of a two-dimensional electron gas (2DEG) in the presence of the Rashba spin-orbit interaction in the diffusive transport regime. When defect scattering is modeled by isotropic short-range potential scatterers the spin Hall conductivity vanishes due to the vertex correction. A nonvanishing spin Hall effect may be recovered for dominantly forward defect scattering.
\end{abstract}

DOI: 10.1103/PhysRevB.70.041303

PACS number(s): 73.23.Ra, 72.25.Dc, 72.20.Dp

Spintronics is the rapidly developing field of research aimed at using not only the charge but also the spin degree of freedom of electrons in electronic circuits and devices. ${ }^{1}$ In order to be compatible with microelectronic technology, effective spin injection into conventional semiconductors is necessary. Injection of spins via attached ferromagnets has turned out to be quite difficult. ${ }^{2,3}$ This is one motivation to investigate the possibilities of making use of the spin-orbit (SO) interaction, which may spin-polarize a nonmagnetic conductor simply by applying a source-drain bias. ${ }^{4-9}$ The two-dimensional electron gas (2DEG) is an ideal model system to investigate the physics of these effects. In sufficiently asymmetric confinement potentials the so-called Rashba term dominates the SO interaction. ${ }^{10}$ The Datta spin transistor concept ${ }^{11}$ is based on the tunability of the Rashba interaction by an external gate potential. ${ }^{12}$

Applying an electric field in the $x$ direction of a Rashba 2DEG spanning the $x, y$ plane induces a charge current in the $x$ direction, and also a homogeneous spin accumulation in the $y$ direction proportional to the field strength. ${ }^{6,7}$ Recently, Sinova et al. reported a persistent spin Hall current ${ }^{9}$ for a ballistic Rashba 2DEG. The acceleration of the electrons by the external electric field (along the $x$ direction) modifies the SO-induced pseudomagnetic field such that the spins are tilted out of the 2DEG plane in directions that are opposite for positive and negative lateral momentum $\left(k_{y}\right)$ states. This corresponds to a flow of $s_{z}=+1 / 2$ and $-1 / 2$ spins in opposite directions without a corresponding net charge transport. ${ }^{9}$ Sinova et al. suggest that the spin Hall current should be rather robust against disorder scattering, which implies that the effect is measurable in Hall bars of mesoscopic dimensions. Note that the ballistic spin Hall effect is quite different from the spin Hall effect reported earlier for diffuse paramagnetic metals, which is caused essentially by impurity scattering. ${ }^{13,14}$ In the weak scattering regime, in which the broadening is smaller than the SO-induced splitting of the energy bands, the lifetime broadening of the self-energy has recently been found to have small effects on the ballistic spin Hall current. ${ }^{15,16}$ In this Rapid Communication we study the effect of disorder on the spin Hall effect in the diffuse re- gime, in which the scattering rate is larger than either the frequency or the inverse sample traversal time, but for weak scattering. By taking into account the vertex correction we find that the spin Hall effect vanishes identically for shortrange impurity scattering.

We model the disorder by randomly distributed isotropic short-range potentials and compute longitudinal and transverse (Hall) conductivities for both charge and spin currents by the Kubo formalism in the Born approximation and in the low temperature limit. The SO interaction is subject to a significant conductivity vertex correction, ${ }^{7}$ which we find here to be decisive for the spin Hall current. The vertex correction appears in such a way that the current operator along the $x$ direction corresponding to the Rashba Hamiltonian $J_{x}=e\left\{(\hbar k / m) \mathbf{1}-\lambda \sigma_{y}\right\}$ is modified by substituting $\lambda$ $\rightarrow \tilde{\lambda}=\lambda+\lambda^{\prime}$. Here $\sigma_{i}(i=x, y, z)$ are the Pauli spin matrices. The correction term $\lambda^{\prime}$ is not necessarily small compared with $\lambda$ and found to be $-\lambda$ in the weak scattering regime. Only without the vertex correction does the spin Hall conductivity tend to $e / 8 \pi$ as predicted by Sinova et al. ${ }^{9}$ Physically, the diffuse scattering represented by the vertex correction efficiently scrambles the precession of spins out of the 2DEG plane induced by the applied electric field such that no net spin Hall current remains. On the other hand, the induced spin accumulation in the $y$ direction is much less sensitive to impurity scattering. ${ }^{7}$ The spin Hall conductivity may persist for long-range, anisotropic defect potentials that correspond to predominantly forward scattering.

The Rashba Hamiltonian in the momentum representation and Pauli spin space reads

$$
H_{0}=\left(\begin{array}{cc}
\left(\hbar^{2} / 2 m\right) k^{2} & i \lambda \hbar k_{-} \\
-i \lambda \hbar k_{+} & \left(\hbar^{2} / 2 m\right) k^{2}
\end{array}\right),
$$

where $k=\sqrt{k_{x}^{2}+k_{y}^{2}}, k_{ \pm}=k_{x} \pm i k_{y}$ with $\mathbf{k}=\left(k_{x}, k_{y}\right)$ the electron momentum in the 2DEG plane, and $\lambda$ parametrizes the tunable spin-orbit coupling. The eigenfunctions and eigenvalues of the Hamiltonian corresponding to periodic boundary conditions are given as 


$$
\begin{gathered}
\phi_{\mathbf{k} s}=\frac{1}{\sqrt{2 L^{2}}} e^{i \mathbf{k} \cdot \mathbf{r}}\left(\begin{array}{c}
i s k \_/ k \\
1
\end{array}\right), \\
E_{k s}=\frac{\hbar^{2} k^{2}}{2 m}+s \lambda \hbar k,
\end{gathered}
$$

respectively, with $s= \pm$, and $L^{2}$ is the area of the 2DEG. The corresponding (free) Green function is denoted as $g_{k s}(z)$ $=1 /\left(z-E_{k s}\right)$ with an energy $z$ on the complex-energy plane.

The disorder is modeled as randomly distributed, identical defects with point scattering potentials that are neither spin dependent nor flip spins

$$
V(\mathbf{r})=V \mathbf{1} \sum_{i} \delta\left(\mathbf{r}-\mathbf{R}_{i}\right),
$$

which gives rise to an isotropic ( $s$-wave) scattering of electrons. The configurational averaged Green function reads

$$
\widetilde{G}(k \pm)=\frac{1}{z-E_{k \pm}-\Sigma_{k \pm}(z)} .
$$

In the Born approximation the self-energy $\Sigma(z)$ is a stateindependent constant:

$$
\Sigma(z)=\frac{n V^{2}}{2 L^{2}} \sum_{k s} g_{k s}(z)
$$

where $n$ is the impurity concentration and $L$ denotes the linear dimensions of the sample. The self-energy at the Fermi energy is related to the scattering lifetime $\tau$ via $|\operatorname{Im} \Sigma|$ $=\hbar / 2 \tau \epsilon_{F}$.

The charge current operator in spin space reads ${ }^{17} J_{x}$ $=e \partial H_{0} / \partial p_{x}=e\left(b k_{x} \mathbf{1}-\lambda \sigma_{y}\right)$ and $J_{y}=e \partial H_{0} / \partial p_{y}=e\left(b k_{y} \mathbf{1}+\lambda \sigma_{x}\right)$ with $b=\hbar / m$. The spin currents are represented by the Hermitian operators 9

$$
J_{\alpha}^{\sigma_{i}}=\frac{\hbar}{4}\left\{v_{\alpha}, \sigma_{i}\right\}=\frac{\hbar}{4}\left\{\frac{\partial H_{0}}{\partial p_{\alpha}}, \sigma_{i}\right\},
$$

where $\quad \alpha=x, y$, and $z$. Thus $J_{x}^{\sigma_{x}}=(\hbar / 2) b k_{x} \sigma_{x}, J_{x}^{\sigma_{y}}$ $=(\hbar / 2)\left(-\lambda 1+b k_{x} \sigma_{y}\right)$ and $J_{x}^{\sigma_{z}}=(\hbar / 2) b k_{x} \sigma_{z}$, whereas $J_{y}^{\sigma_{x}}$ $=(\hbar / 2)\left(\lambda \mathbf{1}+b k_{y} \sigma_{x}\right), J_{y}^{\sigma_{y}}=(\hbar / 2) b k_{y} \sigma_{y}$, and $J_{y}^{\sigma_{z}}=(\hbar / 2) b k_{y} \sigma_{z}$.

The Kubo formula for the longitudinal electrical conductivity can be written

$$
\sigma_{x x}=\frac{\hbar}{2 \pi L^{2}} \operatorname{Tr}\left\langle J_{x} G^{R} J_{x} G^{A}\right\rangle_{\mathrm{av}},
$$

where the superscripts $R$ and $A$ stand for retarded and $a d-$ vanced, respectively (omitted below for brevity), and the trace is taken over wave vectors and band index. We evaluate $\left\langle J_{x} G J_{x} G\right\rangle_{\mathrm{av}}=J_{x}\left\langle G J_{x} G\right\rangle_{\mathrm{av}} \equiv J_{x} K_{x}$ in the ladder approximation that obeys the Ward relation with the self-energy in the Born approximation. This leads to the Bethe-Salpeter equation

$$
K_{x} \approx \widetilde{G} J_{x} \widetilde{G}+\widetilde{G}\left\langle V K_{x} V\right\rangle_{\mathrm{av}} \tilde{G}
$$

$K_{x}=\widetilde{G} \widetilde{J}_{x} \widetilde{G}$ has the same structure as $\widetilde{G} J_{x} \widetilde{G}$, and $^{7}$

$$
\tilde{J}_{x}=e\left(b k_{x} \mathbf{1}+\frac{\tilde{\lambda}}{k}\left(k_{x} \sigma_{z}-k_{y} \sigma_{y}\right)\right),
$$

with $\tilde{\lambda}=\lambda+\lambda^{\prime}$. The vertex correction $\lambda^{\prime}$ is the solution of

$$
\lambda^{\prime}=\frac{n V^{2}}{4 L^{2}} \sum_{k_{1}}\left[b k_{1} \Pi_{k_{1}}^{-}+\left(\lambda+\lambda^{\prime}\right)\left(\Pi_{k_{1}}^{+}+\Pi_{k_{1}}^{+-}+\Pi_{k_{1}}^{-+}\right)\right],
$$

with $\Pi_{k}^{s}=\Pi_{k}^{++}+s \Pi_{k}^{--}$and $\Pi_{k}^{s s^{\prime}}=\widetilde{G}_{k s} \widetilde{G}_{k s^{\prime}}$.

The generalized spin conductivity tensor in Pauli spin space reads

$$
\sigma_{\alpha x}^{\sigma_{i}}=\frac{\hbar}{2 \pi L^{2}} \operatorname{Tr} J_{\alpha}^{\sigma_{i}}\left\langle G J_{x} G\right\rangle_{\mathrm{av}} \sim \frac{\hbar}{2 \pi L^{2}} \operatorname{Tr} J_{\alpha}^{\sigma_{i}} K_{x}
$$

where the vertex function is the same as before. Symmetry tells us that

$$
\begin{aligned}
\operatorname{Tr} J_{x}^{\sigma_{x}} K_{x}= & \frac{e \hbar b}{8} \operatorname{Tr}\left[b k^{2} \Pi^{+}+\tilde{\lambda} k \Pi^{-}\right] \sigma_{x}, \\
\operatorname{Tr} J_{x}^{\sigma_{y}} K_{x}= & \frac{e \hbar b}{8} \operatorname{Tr}\left[b k^{2} \Pi^{+}+(\tilde{\lambda}+\lambda) k \Pi^{-}\right. \\
& \left.+\lambda \tilde{\lambda}\left(\Pi^{+}-\Pi^{+-}-\Pi^{-+}\right)\right] \sigma_{y}, \\
\operatorname{Tr} J_{x}^{\sigma_{z}} K_{x}= & \frac{e \hbar b}{8} \operatorname{Tr}\left[b k^{2} \Pi^{+}+\tilde{\lambda} k \Pi^{-}\right] \sigma_{z}, \\
\operatorname{Tr} J_{y}^{\sigma_{x}} K_{x}= & \frac{e \hbar b}{8} \operatorname{Tr}\left[\tilde{\lambda} k\left(\Pi^{+-}-\Pi^{-+}\right)-\lambda k \Pi^{-}\right. \\
& \left.-\lambda \tilde{\lambda}\left(\Pi^{+}-\Pi^{+-}-\Pi^{-+}\right)\right] \sigma_{y}, \\
\operatorname{Tr} J_{y}^{\sigma_{y}} K_{x}= & -\frac{e \hbar b \tilde{\lambda}}{8} \operatorname{Tr} k\left(\Pi^{+-}-\Pi^{-+}\right) \sigma_{x}, \\
\operatorname{Tr} J_{y}^{\sigma_{z}} K_{x}= & i \frac{e \hbar b \tilde{\lambda}}{8} \operatorname{Tr} k\left(\Pi^{+-}-\Pi^{-+}\right) \mathbf{1} .
\end{aligned}
$$

Because the Green functions depend only on $k$, the angular averages of $k_{x}^{2}$ and $k_{y}^{2}$ are $k^{2} / 2$, and odd terms with respect to $k_{x}$ and $k_{y}$ in the trace of the equations above vanish by symmetry. Without the SO interaction, all matrix elements of $J_{\alpha}^{\sigma_{i}} K_{x}$ vanish except for $J_{x}^{\sigma_{x}} K_{x}, J_{x}^{\sigma_{y}} K_{x}$, and $J_{x}^{\sigma_{z}} K_{x}$. But also these terms become zero after taking the trace. This means that no spin current flows along the external electric field. ${ }^{7}$ Only the spin Hall conductivity $\sigma_{y x}^{\sigma_{z}}$ proportional to $\operatorname{Tr} J_{y}^{\sigma_{z}} K_{x}$ is nonzero, indicating that a spin Hall current along the $y$ direction and polarized in the $z$ direction may exist when an external electric field is applied along $x$, as predicted for the ballistic limit. ${ }^{9}$

The magnitude of the spin Hall effect can be calculated easily by adopting the following approximation for the product of Green functions at the Fermi energy $\epsilon_{F}$, 


$$
\tilde{G}(k s) \tilde{G}(k s) \approx \frac{2 \pi \tau}{\hbar} \delta\left(\epsilon_{F}-E_{k s}\right)
$$

which holds when the energy dependence of the self-energy is weak and the broadening is small compared to the SO energy splitting at the Fermi energy, $|\operatorname{Im} \Sigma| \ll 2 \hbar \lambda k$. Equation (19) implies that in the weak scattering limit only electrons at the Fermi energy contribute to the spin Hall current. Then

$$
\sigma_{y x}^{\sigma_{z}}=-\sigma_{x y}^{\sigma_{z}}=\frac{e \tilde{\lambda}}{8 \pi \lambda}
$$

for $\epsilon_{F}>0$. This agrees with the ballistic result $\sigma_{y x}^{\sigma_{z}}=e / 8 \pi$ by Sinova et al. ${ }^{9}$ except for a factor $\tilde{\lambda} / \lambda=1+\lambda^{\prime} / \lambda$ due to the vertex correction, but is identical to it when the vertex correction $\lambda^{\prime}$ is neglected.

By substituting Eq. (19) into Eq. (11), the vertex correction $\lambda^{\prime}$ is evaluated as $\lambda^{\prime}=-\lambda$, i.e., the spin Hall conductivity vanishes. Equation (19) is equivalent to the weak scattering or strong SO interaction limit. As far as the spin Hall current is concerned, the effect of the impurity vertex correction is thus found to be much more important than that of the impurity self-energy in the Green function treated by Schliemann and Loss ${ }^{15}$ and by Sinitsyn et al. ${ }^{16}$

The ballistic result can be recovered by considering the frequency dependent conductivity

$$
\sigma_{\mu \nu}^{\xi}=\lim _{\omega \rightarrow 0} \frac{Q_{\mu \nu}^{\xi}(\omega)-Q_{\mu \nu}^{\xi}(0)}{-i \omega},
$$

in terms of the correlation function

$$
\begin{aligned}
Q_{\mu \nu}^{\xi}\left(i \nu_{\ell}\right) & =\frac{1}{L^{2} \beta} \sum_{m} \operatorname{Tr}\left[J_{\mu}^{\xi} G\left(i \omega_{m}+i \nu_{\ell}\right) J_{\nu} G\left(i \omega_{m}\right)\right] \\
& =\frac{1}{L^{2}} \operatorname{Tr} J_{\mu}^{\xi} K_{\nu}\left(i \nu_{\ell}\right),
\end{aligned}
$$

with

$$
K_{\nu}\left(i \nu_{\ell}\right)=\frac{1}{\beta} \sum_{m}\left\langle G\left(i \omega_{m}+i \nu_{\ell}\right) J_{\nu} G\left(i \omega_{m}\right)\right\rangle_{\mathrm{av}} .
$$

The vertex correction is calculated as before resulting in

$$
K_{x}\left(i \nu_{\ell}\right)=\frac{1}{\beta} \sum_{m} \widetilde{G}\left(i \omega_{m}+i \nu_{\ell}\right) \widetilde{J}_{x} \widetilde{G}\left(i \omega_{m}\right),
$$

where $\widetilde{J}_{x}$ includes $\tilde{\lambda}=\lambda+\lambda^{\prime}(\omega)$ with

$$
\lambda^{\prime}(\omega) \approx-\frac{\hbar}{\tau-i \hbar \omega+\hbar / \tau},
$$

and letting $i \nu_{\ell} \rightarrow \hbar \omega+i 0$. Here we assumed that $\omega \ll \epsilon_{F}$ and [as in Eq. (19)] weak scattering. ${ }^{18}$ This result generalizes Eq. (20). When the $\tau \rightarrow \infty$ limit is taken first, $\lambda^{\prime}(\omega) \rightarrow 0$, thus recovering the ballistic limit. ${ }^{9}$ When we take the $\omega \rightarrow 0$ limit first, $\lambda^{\prime}(\omega)=-\lambda$, and the spin Hall conductivity vanishes as before.
We made the rather crucial approximation that the scattering potential is short-ranged, thus isotropic in momentum space. As mentioned above, Sinova et al. ${ }^{9}$ explain the ballistic spin Hall current in terms of the precession of spins out of the 2DEG plane when accelerated by the electric field. The extra momentum change is only meaningful for electrons near the Fermi surface, viz., the spin Hall current in the Rashba 2DEG is carried only by electron spins near the Fermi surface. In the presence of isotropic impurity scattering, electrons with momentum $\mathbf{k}$ are scattered into all other momenta $\mathbf{k}^{\prime}$ at the Fermi energy with equal rate, and the spin Hall current disappears with the average spin tilting. This picture is not appropriate anymore when the impurity potentials are long-ranged, scattering predominantly in the forward direction. In that case the short-range model misrepresents the "skew scattering" corresponding to a nonzero Hall angle. ${ }^{19}$ We argue below that the spin Hall conductance reacts more sensitive to randomization than the diagonal conductance $\sigma_{x x}{ }^{20}$

For long-range anisotropic scatterers the longitudinal conductivity is governed not by the energy lifetime $\tau$ but the transport (momentum) lifetime $\tau_{t}$ because the momentum integration in the vertex function over $V^{2} k_{x}$ ( $x$ is the current direction) does not vanish. ${ }^{18}$ Physically this means that the forward (small angle) scattering does not contribute to the resistivity. Without SO interaction, the vertex correction due to anisotropic scattering reads

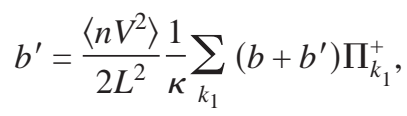

where $b=\hbar / m, 1 / \kappa=\tau / \tau_{1}$, and $\left\langle n V^{2}\right\rangle$ is an average of the scattering potential over the Fermi surface. The transport lifetime is given by $1 / \tau_{t}=1 / \tau-1 / \tau_{1}$.

When the SO interaction is incorporated into this vertex correction, the expression of the longitudinal charge conductivity and spin accumulation obtained before ${ }^{7}$ are modified as

$$
\sigma_{x x}=2\left[\frac{e^{2} \tau_{t}}{m} n_{0}+e^{2} D \tau_{t} \lambda^{2}\right]
$$

and

$$
\left\langle s_{y}\right\rangle=2 \tau_{t} e E D \lambda,
$$

respectively. Here we have used the following relations: $1 / \tau=2 \pi n V^{2} D / \hbar=n V^{2} m / \hbar^{3}$, with $D=m / 2 \pi \hbar^{2}$, where $D$ is the density of states of $2 \mathrm{DEG}$. Note that the relation $\sigma_{x x}^{\uparrow \uparrow}$ $=\sigma_{x x}^{\downarrow \downarrow}$ holds for arbitrary values of $b^{\prime}$ and $\lambda^{\prime}$.

The spin Hall effect may survive when small angle scattering dominates because only states close to each other in momentum space are scrambled. The anisotropy may affect the effective current operator in Eq. (11): the first term in parenthesis on the right-hand side becomes $\lambda$ in the isotropic scattering case and is likely to dominate for not too large long-range potentials. The vertex correction $\lambda^{\prime}$ is then given by 


$$
\lambda^{\prime}=\frac{\left\langle n V^{2}\right\rangle}{4 L^{2}} \frac{1}{\kappa^{\prime}} \sum_{k_{1}} b \Pi_{k_{1}}^{-},
$$

in which $k_{1 x}^{2}$ and $k_{1 y}^{2}=k_{1}^{2}-k_{1 x}^{2}$ are replaced with weighted averages $k_{1}^{2} / 2 \kappa^{\prime}$ and $k_{1}^{2}-k_{1}^{2} / 2 \kappa^{\prime}$ over the angle. With $1 / \kappa^{\prime}$ $=\tau / \tau^{\prime}$, we get $\tilde{\lambda}=\left(\tau / \tau_{H}\right) \lambda$ and $1 / \tau_{H} \equiv 1 / \tau-1 / \tau^{\prime}$. In the isotropic case, $\tau_{H} \rightarrow \infty$ and $\tilde{\lambda} \rightarrow 0$, but in general the spin Hall current is finite. This argument does not take into account the full effects of the anisotropy but demonstrates how the vertex correction for anisotropic scattering affects the spin-Hall conductivity.

Burkov and MacDonald ${ }^{21}$ computed the spin Hall conductivity for the Rashba 2DEG model system with short-range impurity scattering. These authors focus on the dirty limit in which the lifetime broadening exceeds the SO energy splitting, opposite to the clean limit discussed here, but also find a vanishing spin Hall current. A recent numerical study for finite size systems ${ }^{22}$ found that the spin Hall conductivity vanishes when the system size is larger than the localization length. A direct comparison is not straightforward because the scattering strength in the numerical calculations is not weak and the mean free path, localization length, and system size are of the same order of magnitude, whereas we work with a weak scattering approximation and an infinite system size. Still, it appears that our analytical results are not in conflict with the outcome of these simulations.

Murakami et al. ${ }^{8,23}$ developed a theory for the spin Hall currents in hole-doped semiconductors described by the Luttinger Hamiltonian. Separating the spin Hall current into a topologically conserved (intraband) and nonconserved (interband) contribution, ${ }^{23}$ these authors contend that the former, which does not exist in the Rashba $2 \mathrm{DEG}$, is robust against impurity scattering. ${ }^{24}$ The breakdown of the spin Hall current by impurity scattering in the Rashba $2 \mathrm{DEG}$ discussed here would then correspond to the vanishing of the nonconserved part of the spin Hall current. Microscopic calculations for the Luttinger Hamiltonian analogous to the present ones are necessary to unambiguously prove that the topological spin Hall current indeed survives under impurity scattering.

In conclusion, we have examined the effect of impurities on the spin Hall conductivity of a Rashba-split 2DEG and found that the vertex correction (diffuse electron scattering) to the conductivity is essential, causing the spin Hall effect to vanish.

Note added in proof. Recently, we have become aware of a paper by Murakami ${ }^{25}$ that confirms that the spin-Hall current vanishes in the diffuse Rashba 2DEG but that the vertex correction does not affect the spin-Hall current in the Luttinger hole system.

The authors acknowledge fruitful discussions with Allan MacDonald, Jairo Sinova, Shuichi Murakami, and Shoucheng Zhang. This work was supported by the NEDO international project "Nano-scale Magnetoelectronics," Grants-in-Aid for Scientific Research (C) and for Scientific Research in Priority Areas "Semiconductor Nanospintronics" of The Ministry of Education, Culture, Sports, Science, and Technology of Japan, the NAREGI Nanoscience Project, and the FOM Foundation, the DFG (SFB 410), and the DARPA Spins program.
*Electronic address: inoue@ @uap.nagoya-u.ac.jp

${ }^{1}$ S. A. Wolf, D. D. Awschalom, R. A. Buhrman, J. M. Daughton, S. von Molnar, M. L. Roukes, A. Y. Chtchelkanova, and D. M. Treger, Science 294, 1488 (2001).

${ }^{2}$ R. Fiederling, M. Keim, G. Reuscher, W. Ossau, G. Schmidt, A. Waag, and L. W. Molenkamp, Nature (London) 402, 787 (1999).

${ }^{3}$ Y. Ohno, D. K. Young, B. Beschoten, F. Matsukura, H. Ohno, and D. D. Awschalom, Nature (London) 402, 790 (1999).

${ }^{4}$ F. T. Vas'ko and N. A. Prima, Fiz. Tverd. Tela (Leningrad) 21, 1734 (1979) [Sov. Phys. Solid State 21, 994 (1979)].

${ }^{5}$ L. S. Levitov, Yu. V. Nazarov, and G. M. Eliashberg, Zh. Eksp. Teor. Fiz. 88, 229 (1985).

${ }^{6}$ V. M. Edelstein, Solid State Commun. 73, 233 (1990).

${ }^{7}$ J. Inoue, G. E. W. Bauer, and L. W. Molenkamp, Phys. Rev. B 67, 033104 (2003).

${ }^{8}$ S. Murakami, N. Nagaosa, and S-C. Zhang, Science 301, 1348 (2003).

${ }^{9}$ J. Sinova, D. Cultcer, Q. Niu, N. A. Sinitsyn, T. Jungwirth, and A. H. MacDonald, Phys. Rev. Lett. 92, 126603 (2004).

${ }^{10}$ E. I. Rashba, Fiz. Tverd. Tela (Leningrad) 2, 1224 (1960) [Sov. Phys. Solid State 2, 1109 (1960)]; Yu. A. Bychkov and E. I.
Rashba, Pis'ma Zh. Eksp. Teor. Fiz. 39, 66 (1984) [JETP Lett. 39, 78 (1984)].

${ }^{11}$ S. Datta and B. Das, Appl. Phys. Lett. 56, 665 (1990).

${ }^{12}$ J. Nitta, T. Akazaki, H. Takayanagi, and T. Enoki, Phys. Rev. Lett. 78, 1335 (1997).

${ }^{13}$ J. E. Hirsch, Phys. Rev. Lett. 83, 1834 (1999).

${ }^{14}$ S. Zhang, Phys. Rev. Lett. 85, 393 (2000).

${ }^{15}$ J. Schliemann and D. Loss, Phys. Rev. B 69165315 (2004).

${ }^{16}$ N. A. Sinitsyn, E. M. Hankiewicz, W. Teizer, and J. Sinova, condmat/0310315.

${ }^{17}$ L. W. Molenkamp, G. Schmidt, and G. E. W. Bauer, Phys. Rev. B 64, 121202(R) (2001).

${ }^{18}$ G. D. Mahan, Many-Particle Physics (Kluwer Academic, New York, 2000).

${ }^{19}$ C.-N. Chazalviel, Phys. Rev. B 11, 3918 (1975).

${ }^{20}$ A. G. Mal'shukov and K. A. Chao, Phys. Rev. B 61, R2413 (2000).

${ }^{21}$ A. A. Burkov and A. H. MacDonald, cond-mat/0311328.

${ }^{22}$ Ye Xiong and X. C. Xie, cond-mat/0403083.

${ }^{23}$ S. Murakami, N. Nagaosa, and S-C. Zhang, cond-mat/0310005.

${ }^{24} \mathrm{~S}-\mathrm{C}$. Zhang (private communication).

${ }^{25}$ S. Murakami, cond-mat/0405001. 